\title{
Determination of MS Location through Building Using AoA Method of Frequency $47 \mathrm{GHz}$
}

\author{
Andrita Ceriana Eska ${ }^{1}$
}

\begin{abstract}
This research discusses the determination of mobile station (MS) location of the uplink communication system. The location determination mobile station is based on the angle-of-arrival (AoA) method. The communication propagation is influenced by building environment. The building environment was modeled with diffraction method. Several diffraction methods were used such as single knife edge, and multiple knife edge method. The communication frequency used was $47 \mathrm{GHz}$. The analysis used percentage value at coverage area and comparison of error percent values between two method to determine mobile station location. The percentage of the communication coverage area obtained was $71.4 \%$ or of 255 from 300 nodes. The comparison methods used for mobile station location determination were the selection of the best SNR and localization technique. The error percentage value based on the selection of the best SNR method is $0.95 \%$. The error percentage value based on localization technique method is $0.78 \%$.
\end{abstract}

Keywords—single knife edge, multiple knife edge, bergedung, angle-of-arrival.

\section{INTRODUCTION}

Currently there are many millimeter wave utilizations for communication systems. This is evidenced by a variety of studies that have evolved to use high frequency, such as research on measuring $60 \mathrm{GHz}$ millimeter wave frequency propagation using broadband voice channel [1], and also research for cellular propagation using frequencies of $38 \mathrm{GHz}$ and $60 \mathrm{GHz}$ [2]. The utilization of these frequencies causes atmospheric attenuation due to the influence of water vapor and oxygen, as regulated by atmospheric attenuation standards based on ITU-R in atmosphere [3].

In mobile communication systems, the existence of a building height between Base Transceiver Station (BTS) and Mobile Station (MS) can affect communication. The existence of such buildings can have a variety of sizes, including the height. A study utilized the existence of a building or building in its testing [2]. Other studies performed broadband millimeter wave propagation measurements for urban cellular communications [4], estimates using Angle-of-Arrival (AoA) on the $60 \mathrm{GHz}$ frequency communication path [5], Angle-ofDeparture (AoD) and AoA estimates using mmWave FD MIMO system [6], and accuracy of TDoA and AoA methods of localization using two stations [7].

This paper presents the use of BTS to determine the location of MS based on AoA. Communication system is formed in uplink condition between MS with BTS using communication frequency $47 \mathrm{GHz}$. Given the high frequency

\footnotetext{
${ }^{1}$ Universitas Jember, Jalan Kalimantan 37 Kampus Tegalboto Jember 159 INDONESIA (e-mail: andritacerianaeska@gmail.com)
}

utilization for the growing communication and the need for increased data rates, the utilization of the millimeter wave frequency is still worth investigating. Communication propagation is formed in a state of unobstructed buildings or buildings with varying heights. The MS trajectory is on a 300meter road model. The existence of these trajectories adjacent to the existence of buildings or buildings. Methods to calculate the influence of multiple buildings or multi-building is the method of diffraction single knife edge and multiple knife edge. The frequency used in the communication is 47 GHz. This high frequency can be affected by atmospheric attenuation. In this paper, two BTSs are used to determine the presence of MS. The analysis in this study involves the utilization of macrodiversity from two base stations for the determination of MS locations. Comparison of methods for determining the location of this MS is the first to use the best SNR selection between the two base stations. The second uses localization techniques. Several results are indicated by the percentage ratio of the coverage area of the communication between the two methods and the comparison of the percentage error values between the two methods.

\section{Methodology}

Communication system modeling used is the relationship between the BTS and the MS. The determination of the MS location is determined using the AoA method. The mechanism of communication propagation is modeled by the environment of the building. Single knife edge and multiple knife edge methods are used to determine the propagation of such communications. The communication frequency used is 47 GHz. The utilization of $47 \mathrm{GHz}$ frequency which is a millimeter wave for communication system continues to grow. This high frequency can be affected by atmospheric attenuation. Some of the atmospheric attenuation is the influence of water vapor density and oxygen. Fig. 1 shows atmospheric attenuation [3], with air pressure characteristics of $1.013 \mathrm{hPa}$, temperature $15^{\circ} \mathrm{C}$, and water vapor density of $7.5 \mathrm{~g} \mathrm{~m}$-3. Equation (1) consists of, $\gamma_{a}$, the atmospheric attenuation specification $(\mathrm{dB} / \mathrm{km})$, d, is the Line-Of-Sight (LOS) distance $(\mathrm{km})$. Equation (2) shows the value of $\gamma_{a}$, which consists of $\gamma_{w}$ and $\gamma_{w}$ with $\gamma_{w}$ is water vapor attenuation, whereas $\gamma_{o}$ is oxygen attenuation.

$$
\begin{aligned}
& A=\gamma_{a} d \\
& \gamma_{a}=\gamma_{o}+\gamma_{w} .
\end{aligned}
$$

The condition of the communication environment is modeled by the existence of buildings, BTS, and MS. Macrocell BTS is made for the widest range of communication propagation. Communication system used is 
in uplink conditions, with MS transmit power used for 1 watt. Equation (3) is the noise value $(\mathrm{N})$, which is formed from the Boltzman (K) constant, bandwidth (B), standard noise temperature (To), and noise figure (F) [8]. The noise figure used is $7 \mathrm{~dB}$ and the bandwidth used is $200 \mathrm{MHz}$.

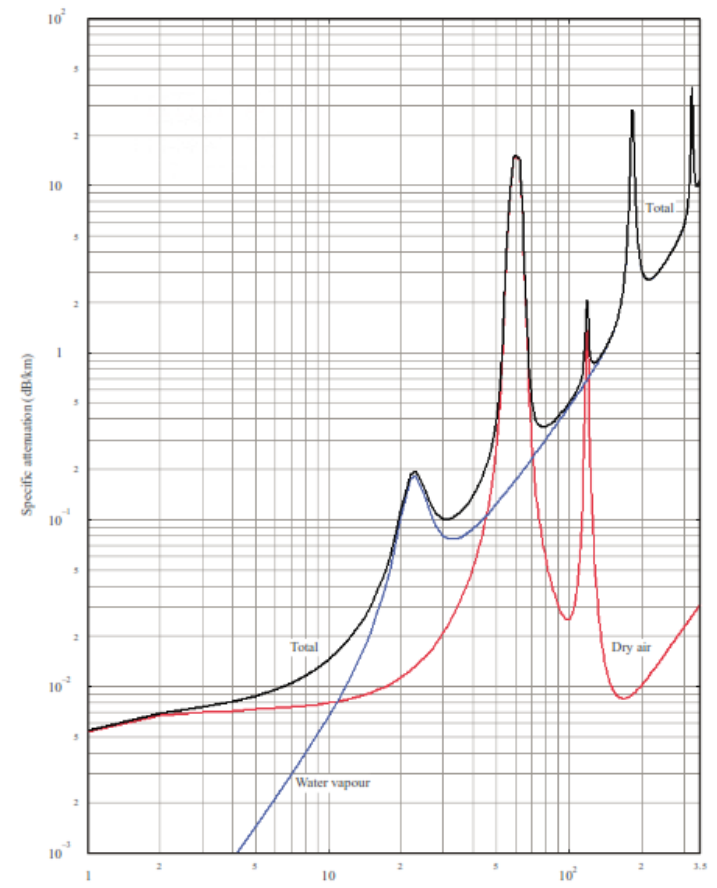

Fig. 1 Characteristics of atmospheric attenuation.

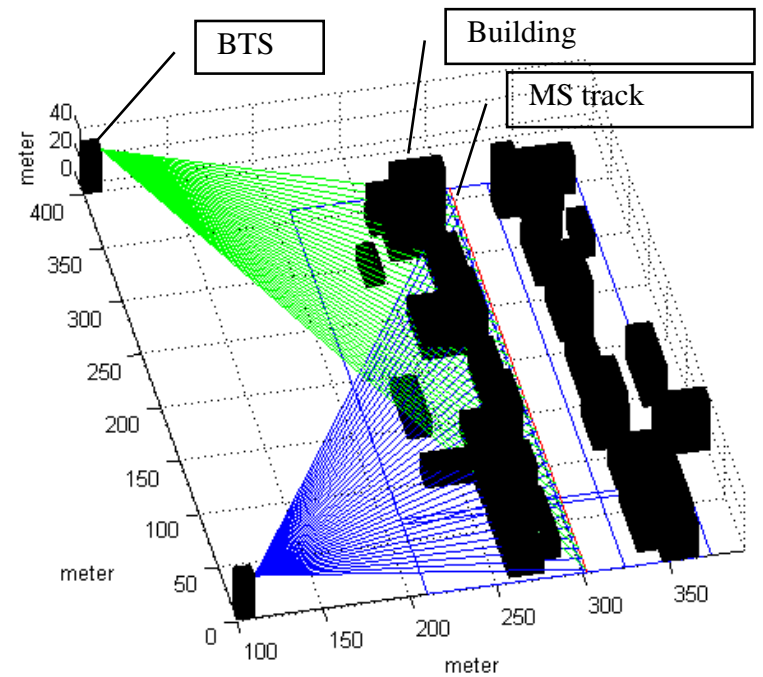

Fig. 2 MS trajectory propagation model with BTS.

$$
N=k T_{o} B F .
$$

The calculation of pathloss value refers to (4). The equation is the Friis equation, which consists of several values, such as the gain of the transmitting and receiving antennas [8]. L represents loss, GT is the gain transmitter $(\mathrm{dB})$, GR is the gain receiver $(\mathrm{dB}), \mathrm{d}$ represents the communication distance, and $\lambda$ is the wavelength (m). At (5) the signal-to-noise-ratios (SNR) is shown in $\mathrm{dB}$ and $\mathrm{s}$ is the signal, also in $\mathrm{dB}$.

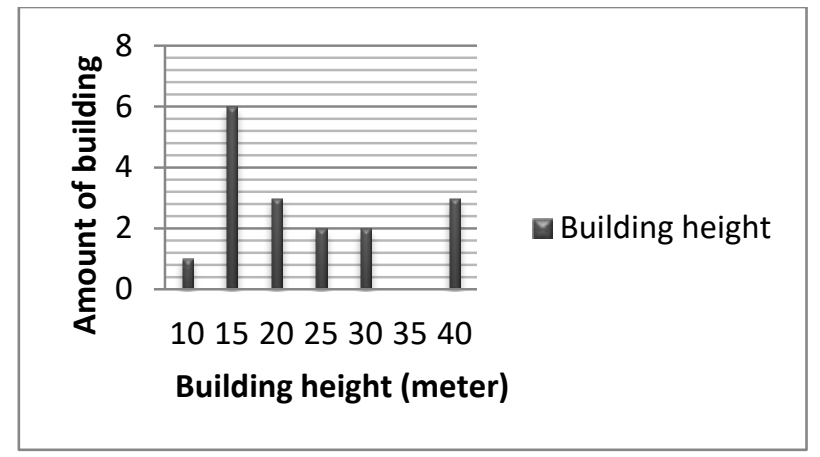

Fig. 3 Variation of building height.

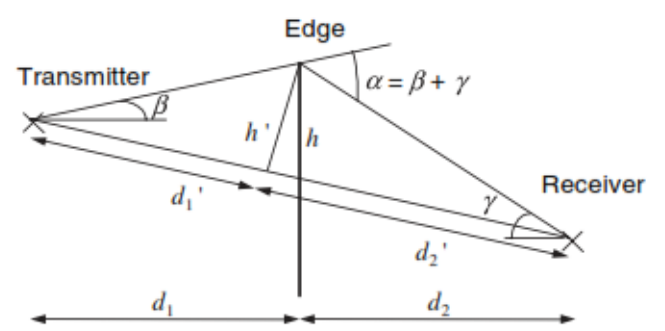

Fig. 4 Difraksi knife edge.

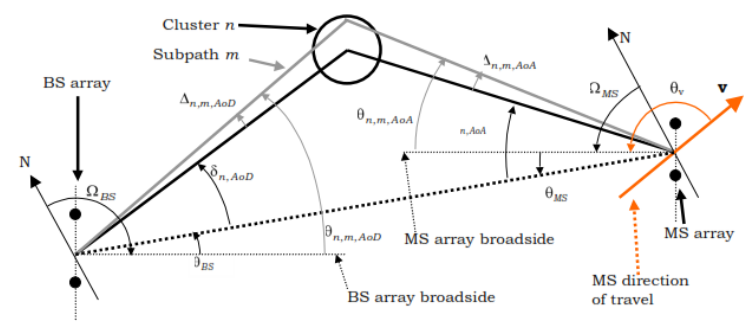

Fig. 5 Parameter sudut antara BS dengan MS.

$$
\begin{aligned}
& L=G_{T} G_{R}\left(\frac{\lambda}{4 \pi d}\right)^{2} \\
& S N R=\frac{S}{N} .
\end{aligned}
$$

Fig. 2 shows the modeling of a woven environment. The building variations used for environmental modeling can be shown in Fig. 3. The width of the road is modeled as far as 24 meters. The width of the road consists of the following two streets with the presence of sidewalks on both sides. The height of the BTS is modeled as high as 30 meters. The MS height is modeled at a height of 1.5 meters, while the distance between the BTS is modeled as far as 400 meters.

The propagation mechanism of multi-purpose or multibuilding communication can be modeled using the single knife edge and multiple knife edge diffraction methods, as in Fig. 4 [8]. Equation (6) shows the calculation of the effect of the knife edge diffraction. Furthermore, the results are taken into account with Fresnel zones. Parameter $\mathrm{v}$ is a FresnelKirchhoff diffraction parameter, $\lambda$ is the wavelength $(\mathrm{m}), \mathrm{h}$ is the height of the diffraction point (m), also $d_{1}$ and $d_{2}$ is the distance between the transmitter or receiver to the diffraction point (m) [9]. 


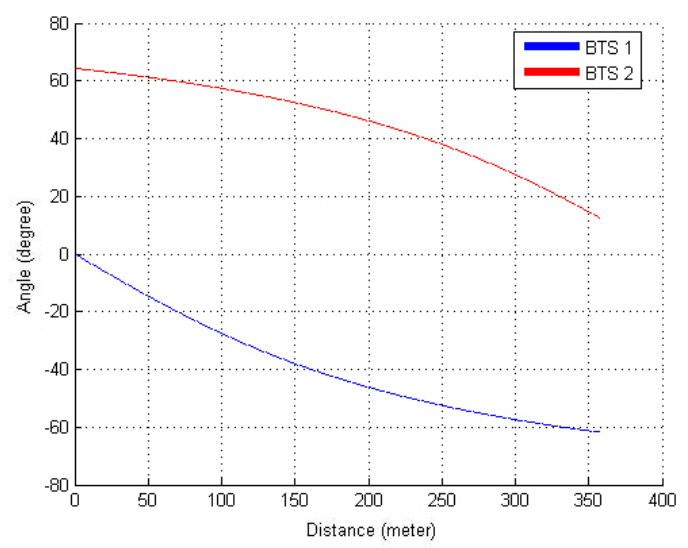

Fig. 6 AoA pada BTS 1 dan BTS 2.

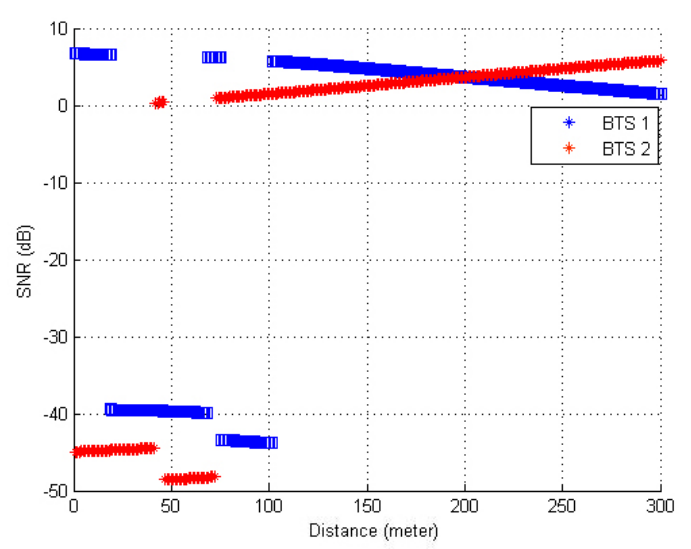

Fig. 7 SNR uplink condition passes a multi-purpose building block frequency of $47 \mathrm{GHz}$.

$$
v=h \sqrt{\frac{d\left(d_{1}+d_{2}\right)}{\lambda d_{1} d_{2}}}=\alpha \sqrt{\frac{2 d_{1} d_{2}}{\lambda\left(d_{1}+d_{2}\right)}} .
$$

The AoA mechanism is shown in Fig. 5. In the picture indicated angle parameter of downlink and uplink condition between BTS with MS, with $\Omega_{\mathrm{ms}}$ is an angle between LOS BS or BTS and MS. MS broadside $\delta_{\mathrm{n}, \mathrm{AOA}}$ is $\mathrm{n}(1 \ldots \mathrm{N})$ path for LOS AoA $\theta_{0, \mathrm{MS}}$. and $\mathrm{v}$ is velocity vector [10]. Research conducted focuses on uplink conditions with propagation barriers in the form of variations of buildings that have varying heights.

\section{RESULTS}

This section discusses the results of the study. The research undertaken focuses on determining the MS locations based on the angle of the AoA. SNR values are obtained from the propagation results of uplink communication conditions with multi-building blocks and atmospheric attenuation. The multibuilding obstacles in this study were analyzed using the knife edges and multiple knife edges.

The results are shown in Fig. 6. The figure shows an AoA angle formed from each MS movement on the MS path. The MS trajectory is 300 meters or 300 points. Each point is a communication point between BTS and MS. AoA values form the basis for MS location determination. The SNR value is shown in Fig. 7. SNR value of BTS 1 is shown in blue, while SNR of BTS 2 is shown in red. SNR values seem to be complementary when SNR decreases between BTS 1 and BTS 2. Techniques macrodiversity using BTS more than one can help overcome the decrease in SNR value on other BTS. The analysis presented in this research is to determine the location of the MS using two methods. The first method is the location of the MS based on the best SNR between the two base stations, and the second method is the localization technique.

In Fig. 8, it is indicated the location of the MS using the first method and the second method. The MS positioning results for the first method are shown in blue, while the second method is shown in red. In the picture, it can be seen several dots of blue and red color overlap each other. Some of the data show the MS travel is 10 meters, the location of the MS using the first method is 307.78 meters and using the second method is 307.78 meters. When the MS travels 80 meters, the location of the MS using the first method as far as 304.26 meters and second method as far as 304,26 meters. From the data, there is a similarity in the MS location determination. Several points do not connect each other, between the blue dots with red dots, it indicates the distance or location of the MS using the first method. The second method has a value difference that is quite high. Some of the data are when the MS reaches 180 meters. The location of the MS using the first method is 303.6 meters. The second method is 303.69 meters. When MS travels as far as 290 meters, the location of the MS using the first method is 304.11 meters. The second method is 303.73 meters.

More detail determining MS location is shown in Fig. 9. It appears that some of the data have distances that are relatively close such as when the MS travels 10 meters, indicating the location of the MS using the first method of excess distance of 5.79 meters and the second method of the excess distance of 5.79 meters. When the MS travels 80 meters, the location of the MS using the first method of excess distance is 4.4 meters. The second method of excess distance of 4.4 meters. Some data show relative further distance between the first method and the second method, when the MS travels 180 meters, the location of the MS uses the first method of the excess distance of 2.19 meters. The second method of the excess distance is 1.7 meters. When the MS travels 290 meters, the location of the MS using the first method of excess distance is 3.82 meters. Furthermore, the second method of the excess distance is 2.1 meters.

Fig. 10 shows the percent error value of the excess distance from the actual MS location, using the first method and the second method. For more details, see Fig. 11. The figure shows some data when the distance from BTS 1 and BTS 2 is relatively close, for example when the MS travel is 10 meters, using the first method, it is obtained the percentage error of $1.92 \%$ while with the second method, it is obtained the percentage error of $1.92 \%$. When the MS travel 80 meters, with the first method it is obtained the percentage error of $1.45 \%$, while with the second method it is obtained the percentage error of $1.45 \%$. The other data shows that the distance of BTS 1 and BTS 2 is relatively far. When the MS 
journey 180 meters, with the first method it is obtained error of $0.73 \%$, while the second method of error percentage of $0.57 \%$. When the MS travel s 290 meters, the first method generates an error of $1.27 \%$, while the second method gives an error of $0.69 \%$.

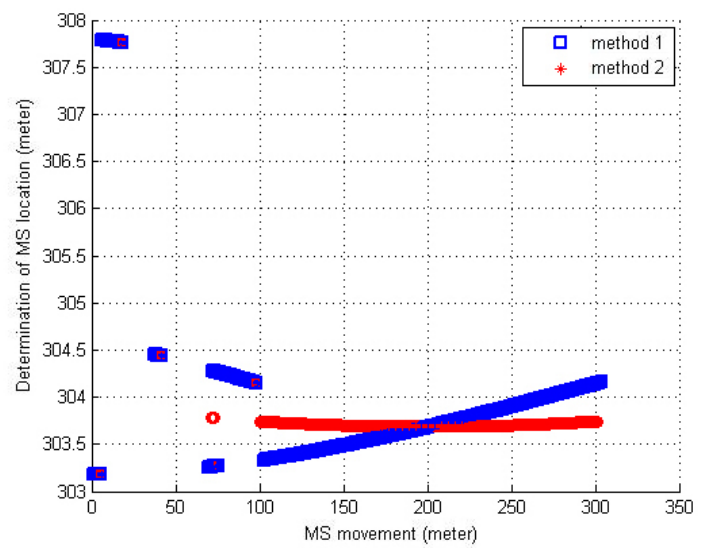

Fig. 8 Results of the determination of MS location using method 1 and 2.

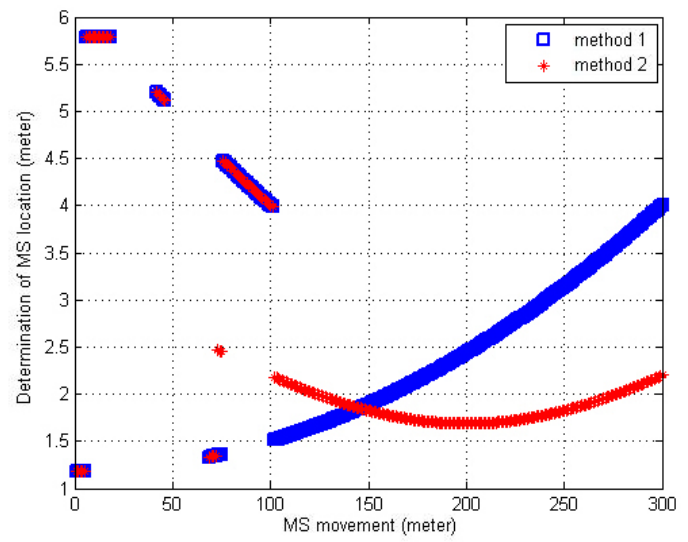

Fig. 9 The distance over the MS location should be.

\section{Discussion}

This section discusses the test results from the MS location determination. The communication frequency used is $47 \mathrm{GHz}$. The utilization of such frequencies can be affected by atmospheric attenuation. Communication process used is in uplink condition. The environment of communication propagation is faced with the existence of buildings. MS moves on track of 300 meters or to 300 points. As a result, the BTS 1 shows the number of points of 225 points or $75 \%$, and on BTS 2 with 233 points or $77.67 \%$.

The fully building conditions were analyzed using single knife edge and multiple knife edge diffraction methods. The location of the MS is based on the angle of AoA. Two BTSs were used for MS location determination. The macrodiversity of both BTSs has an effect on the improvement of the SNR. The determination of the location of the MS was done by comparing two methods, namely the first method of selecting the best SNR value between the two base stations, and the second method using the localization technique. Results of the area covered from both base stations generate 255 points from 300 points or $71.4 \%$. Differences from both methods can be considered from the percentage of error. The average error value of the first method is $0.95 \%$, while the average error value of the second method is $0.78 \%$, thus it is shown that the method using localization technique in determining the MS location is better.

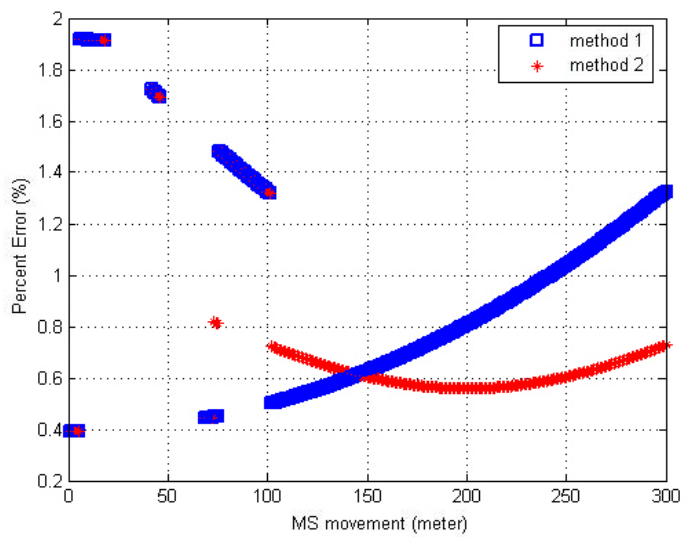

Fig. 10 The percentage error value of excess distance from the MS location should be.

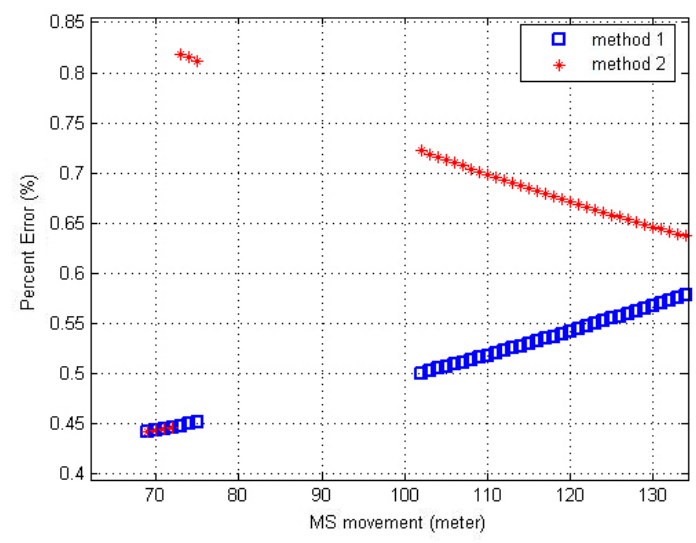

Fig. 11 The percentage value of excess distance error when distance from base stations 1 and base 2 is relatively close together.

\section{CONCLUSION}

The determination of the MS location is based on AoA. The MS moves on track of 300 meters. The communication environment is modeled in the presence of buildings. The modeling of the buildings is formed with different heights and altitudes. The diffraction mechanisms used for the environment are single knife edges and multiple knife edges. The communication frequency used is $47 \mathrm{GHz}$. In communication systems, the use of such frequencies can be affected by the atmospheric attenuation. For location determination MS used two BTS. The role of macrodiversity of both base stations is used to maintain communication. For the determination of the location of MS, it is used the comparison of two methods, namely the method of selecting the best SNR and methods of localization techniques. In the best SNR selection method, from the two base stations, the 
average error rate is $0.95 \%$, while the localization technique method gives an average error rate of $0.78 \%$. Thus, methods that utilize localization techniques result in the determination of the MS locations with better error values.

\section{REFERENCES}

[1] E.B. Dor, T.S. Rappaport, Y. Qiao, dan S.J. Lauffenburger, "Millimeter-wave $60 \mathrm{GHz}$ Outdoor and Vehicle AOA Propagation Measurements using a Broadband Channel Sound,” IEEE Globecom, 2011.

[2] T.S.Rappaport, E.B.Dor, J.N.Murdock, dan Y.Qiao, “38 GHz and 60 $\mathrm{GHz}$ Angle-dependent Propagation for Cellular \& Peer-to-Peer Wireless Communications," IEEE ICC Wireless Communications, 2012.

[3] ITU, ITU-R Radio Communication Sector of ITU (Attenuation by atmospheric gases), ITU-R P.676-10, Geneva: Electronic Publication, 2013.

[4] T.S Rappaport, F. Gutierrez, E.B Dor, J.N. Murdock, Y. Qiao, dan J. I. Tamir, "Broadband Millimeter-Wave Propagation Measurements and Models Using Adaptive-Beam Antennas for Outdoor Urban Cellular Communication,” IEEE Transactions on Antennas and Propagations, Vol.61, No.4, 2013.

[5] Y.M. Tsang, dan A.S.Y. Poom, "Successive AoA Estimation : Revealing the Second Path for $60 \mathrm{GHz}$ Communication System," IEEE, Forty-Ninth Annual Allerton Conference, 2011.

[6] D.Zhu, J.Choi, dan R.W.Heath, "Auxiliary Beam Enabled AoD and AoA Estimation in MMWave FD-MIMO Systems,” IEEE, 2016.

[7] J.Yin, Q.Wan, S.Yang, dan K.C.Ho, "A Simple and Accurate TDOA-AOA Localization Method Using Two Stations,” IEEE Signal Processing Letters, Vol. 23, 2016.

[8] J.S. Seybold, Introduction to RF Propagation, New Jersey : John Wiley\&Sons, 2005.

[9] S.R. Saunders, dan A.A. Zavala, Antennas and Propagation for Wireless Comunication Systems second edition, England : John Wiley \& Sons, 2007.

[10] 3GPP $3^{\text {rd }}$ Generation Partnership Project Technical Specification Group Radio Access Network Spatial Channel model Multiple Input Multiple Output (MIMO) simulations, 3GPP, 2011. 